\title{
OPEN Association between magnesium concentrations and prediabetes: a systematic review and meta-analysis
}

\author{
Sara Ebrahimi Mousavi, Seyed Mojtaba Ghoreishy, Amirhossein Hemmati \& \\ Hamed Mohammadi ${ }^{\bowtie}$
}

Studies on the association between serum magnesium level and prediabetes yielded inconsistent results. Therefore, the present meta-analysis was designed to examine the association between serum magnesium levels and prediabetes. Online databases including PubMed, Embase, Scopus and Google Scholar were searched up to October, 2020. A total of 10 studies that reported mean and standard deviation (SD) of magnesium levels in prediabetes and healthy control group were identified. Random effects models were used to pool weighted mean differences (WMDs) of serum magnesium levels. Pooled-analysis showed that subjects with prediabetes had significantly lower serum magnesium levels compared with healthy controls (WMD $=-0.07 \mathrm{mmol} / \mathrm{L} ; 95 \% \mathrm{Cl}-0.09,-0.05 \mathrm{mmol} / \mathrm{L}, \mathrm{P}<0.001$ ). A significant heterogeneity observed across included studies $\left(I^{2}=95.6 \%, P<0.001\right)$. However, different subgroup analysis did not detect the potential source of observed heterogeneity. Withdrawal of each individual study had no effect on the overall results. The present meta-analysis showed that circulating magnesium levels in people with prediabetes were significantly lower than healthy controls, confirming that magnesium deficiency may play a role in development and progression of prediabetes. Further studies with larger sample size and robust design are warranted to confirm present results.

Prediabetes is defined as an intermediate state of hyperglycemia with glycemic parameters between normal and diabetes threshold ${ }^{1}$. To date, no laboratory threshold has been agreed for the diagnosis of prediabetes. According to the American Diabetic Association (ADA), prediabetes is defined as impaired fasting glucose (IFG) of $5.6-6.9 \mathrm{mmol} / \mathrm{L}$ and/or $2 \mathrm{~h}$ post-challenge glucose of $7.8-11.0 \mathrm{mmol} / \mathrm{L}$ with a $75 \mathrm{~g}$ oral glucose tolerance test (impaired glucose tolerance [IGT]) or based on a HbAlc value of 5.7-6.4\% ${ }^{2}$. However, the World Health Organization (WHO) has set a prediabetes threshold of IFG 6.1-6.9 $\mathrm{mmol} / \mathrm{L}^{3}$. The lower IFG threshold in the ADA criterion is due to the increased risks of micro- and macro-vascular complications near this threshold ${ }^{4}$. ADA criteria overestimates the prevalence of prediabetes and include more people who are at higher risk for diabetes and cardiovascular disease ${ }^{5,6}$. It has been suggested that individuals with glucose metabolic disorders had altered mineral metabolism ${ }^{7}$. In this regard, magnesium has a crucial role in development and progression of chronic disorders such as type 2 diabetes and cardiovascular diseases ${ }^{8-10}$. Magnesium is an important cofactor in many biochemical reactions and also plays a role in regulating a number of vital functions such as muscle contraction, neuromuscular conduction, glucose control, myocardial electrical activity and blood pressure ${ }^{11-14}$. Recently, two meta-analysis have shown a beneficial role of magnesium supplementation on glucose parameters and insulin sensitivity in people with or at risk of diabetes ${ }^{15-17}$.

Previous studies that investigated the associations between the circulating levels of magnesium and prediabetes have yielded inconsistent results. Some studies documented the lower levels of magnesium in people with prediabetes compared to their healthy controls ${ }^{18-20}$, while others did not find significant differences ${ }^{21,22}$. Till now, there is no meta-analyses to address these inconsistent results. Therefore, the present systematic review and meta-analysis was designed to quantitatively examine the association between circulating magnesium levels and prediabetes. 


\section{Methods}

This study was performed based on the PRISMA (Preferred Reporting Items for Systematic Reviews and MetaAnalyses) protocol for reporting systematic reviews and meta-analyses ${ }^{23}$.

Search strategy. Online databases including Medline, Embase, Scopus and Google scholar were searched up to October 2020, without limitation in publication time and language. The following key words were used in the current study: "Magnesium" AND "Prediabetic state" OR "Impaired glucose tolerance" OR "prediabetes" OR "Prediabetes" OR "Hyperglycaemia" OR" Borderline diabetes". In addition, we manually checked all reference lists of included articles and related reviews ${ }^{24,25}$ to avoid missing any relevant studies (Supplementary Table 1).

Inclusion and exclusion criteria. All original observational case-control and cross-sectional studies were included if they: (1) examined the levels of magnesium in prediabetes individuals; and (2) provided sufficient data on serum/plasma magnesium levels in both prediabetes and control groups. papers were excluded if they: (1) enrolled patients with a disease other than prediabetes (2) without healthy control group, (3) published in non-English language or reported duplicate data; and (4) were reviews, conference abstract, clinical trials, letters, editorial articles, or case reports.

Data extraction. Two independent investigators (SEB and SMG) extracted the relevant data and third investigator (HM) resolve any disagreements. The following information were extracted: author, publication year, country, number of cases and controls, mean age, criteria of prediabetes, magnesium levels (mean $\pm S D)$, magnesium assessment method, and study design.

Quality of assessment. To evaluate the quality of the case-control studies, we used the Newcastle-Ottawa Scale (NOS) to evaluate the following characteristics with a maximum of nine starts for each study ${ }^{26}$.

A. Selection (4 items): adequacy of case definition; representativeness of the cases; selection of controls; and definition of controls.

B. Comparability ( 1 item): comparability of cases and controls on the basis of the design or analysis. According to the confounders including age, sex, the comparability of included studies was assessed. If a study adjusted its results for age, one star was given to it. Also, two stars were given to a study which adjusted its findings for age and sex.

C. Exposure (3 items): ascertainment of exposure; same method of ascertainment for cases and controls; and non-response rate (same rate for both groups).

Also, the evaluation of the quality of cross-sectional studies with the modified version of NOS was performed by evaluating the following characteristics with a maximum of ten starts for each study ${ }^{27}$.

A. Selection (4 items): representativeness of samples; sample size; non-respondents characteristics; ascertainment of the exposure. To assess exposure, if a study has used the validated method, it receives two stars and if it is not validated, it receives one star.

B. Comparability ( 1 item): comparability of two groups on the basis of the design or analysis. According to the confounders including age, sex, the comparability of included studies was assessed. As in the case-control studies, it received one star if the study results were controlled for age only, and two stars if adjusted for age and sex.

C. Outcome (3 items): assessment of the outcome: statistical test. To evaluate the result, if a study was evaluated using a record linkage or independent blind evaluation, it received two stars and, in the absence of a description, one star.

Articles with a total score of $0-4,5-7$, and 8-10 were considered as low, moderate and high quality, respectively.

Statistical analysis. Statistical analysis was performed by using Stata 14.0 and $\mathrm{P} \leq 0.05$ was considered statistically significant. The overall relationship between serum and plasma magnesium levels and prediabetes was calculated by comparing the mean and standard deviation (SD) of magnesium levels in prediabetes compared with the healthy control group. When a study provided standard error (SE), SD was calculated by using this equation: ( $\mathrm{SD}=\mathrm{SE} \times$ square root [number of participants]). Also, if a study reported medians and ranges or $95 \%$ CIs, we computed mean (SD) by Hozo's method ${ }^{28}$. Prior the analysis of effect size, the levels of magnesium in serum and plasma were converted to $\mathrm{mmol} / \mathrm{L}$. Weighted mean differences (WMDs) with 95\% CIs in magnesium levels were estimated by a random-effects model ${ }^{29}$. Heterogeneity between included studies was assessed by Cochran's Q test and the $\mathrm{I}^{2}$ statistic $^{30}$. Subgroup analysis was performed to find a possible source of heterogeneity. In order to evaluate the impact of each individual study on the overall effect, sensitivity analysis was applied. We used Begg's test and Egger's liner regression test to evaluate publication bias ${ }^{31}$. In addition, we performed a trim-and-fill approach to obtain an adjusted effect size that takes into account publication bias ${ }^{32}$. 


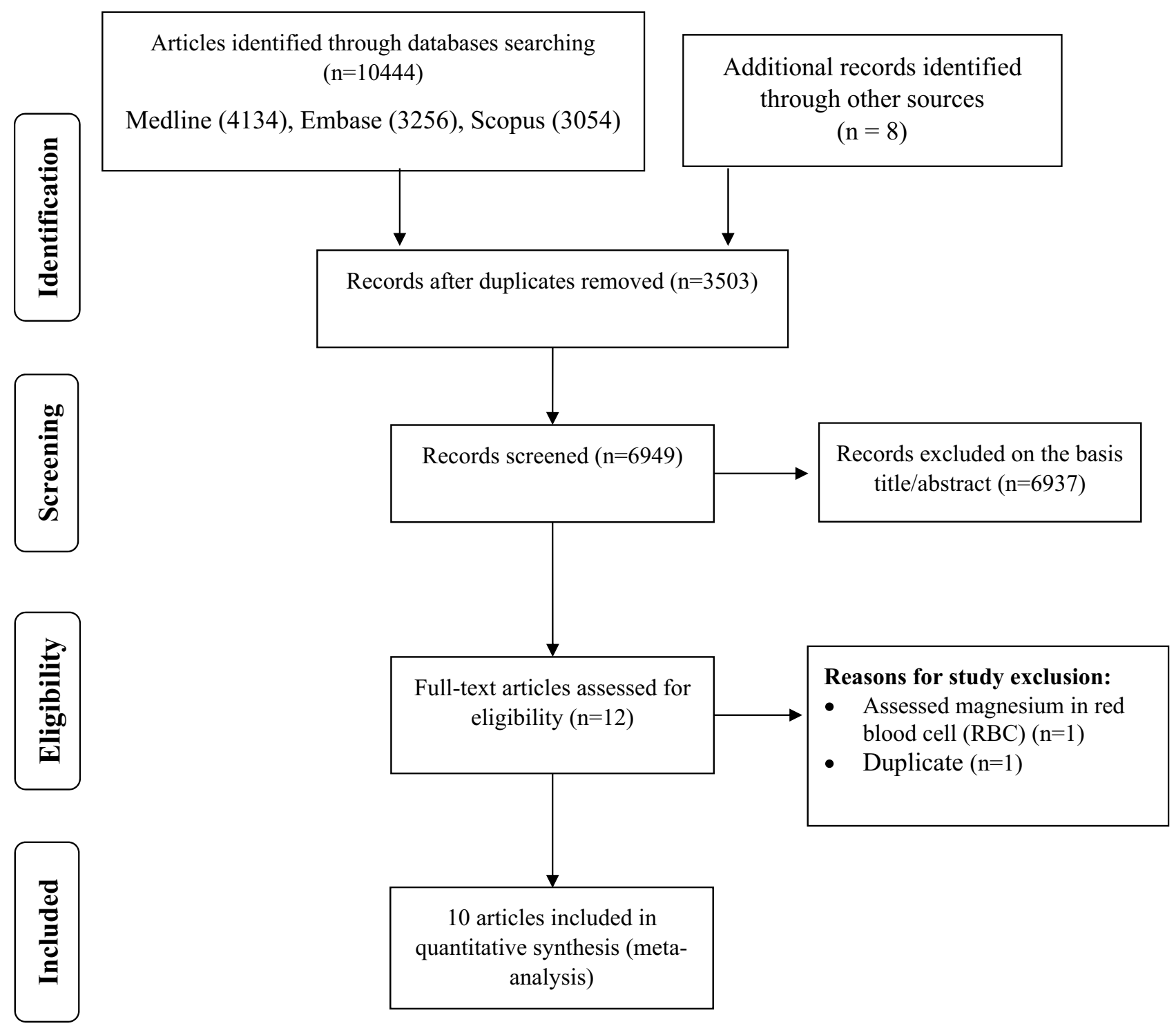

Figure 1. PRISMA flowchart describing the study's systematic literature search and study selection.

\section{Results}

Study selection. In our primary search 10,452 articles identified from above mentioned databases. After removing 3503 duplicates, 6949 articles retrieved for screening. Based on initial title and abstract screening, we excluded 6933 articles because of the following reasons: unrelated studies $(n=5525)$, animal studies $(n=1147)$, review articles $(n=251)$ and non-English studies $(n=14)$. By examining the remaining 12 full-text articles, we exclude one study because it assessed the magnesium levels in red blood cell (RBC) ${ }^{33}$. In addition, one similar data was published twice ${ }^{18,34}$ and we included the article that report data in detail and extractable form ${ }^{18}$. Eventually, ten observational studies met our eligibility criteria and include in final analysis (Fig. 1 and Supplementary Table 1$)^{18-22,35-39}$.

Study characteristics. Characteristics of eligible studies are presented in Table 1. Included studies were published between 1990 and 2019 and the total sample size was 13,455 subjects (2979 prediabetes patients and 10,476 healthy controls). The criteria for diagnosing prediabetes in most studies were criteria recommended by the $\mathrm{ADA}^{35,38,39}$ and $\mathrm{WHO}^{19,37}$. Of total included studies, one study only enrolled males ${ }^{20}$, while the other studies included both genders. The mean age of all subjects ranged between 29 and 67 years. Out of 10 studies, 3 studies were conducted in China ${ }^{18,19,36}$ and others were conducted in the United states ${ }^{21}$, Netherlands ${ }^{37}$, Sweden ${ }^{20}$, Bangladesh $^{22}$, India ${ }^{39}$, Italy ${ }^{38}$ and Turkey ${ }^{35}$. Two studies assessed magnesium level by inductively coupled plasma spectrometry ${ }^{18,19}$, two studies used atomic absorption ${ }^{20,36}$ and in other studies other methods were used. Six studies used cross-sectional design ${ }^{20,21,35,37-39}$ and the rest of them applied case-control design. The results of Newcastle-Ottawa scale were presented in Table 2. Among ten publications included in the systematic review six studies assigned as a high quality (NOS $=7-9)$ and four studies assigned as a moderate quality $(\mathrm{NOS}=6)$. 


\begin{tabular}{|c|c|c|c|c|c|c|c|c|c|c|}
\hline $\begin{array}{l}\text { First author } \\
\text { (year; location) }\end{array}$ & Study design & Sample & \multicolumn{2}{|c|}{ Population and sample size } & \multirow{3}{*}{\begin{tabular}{|l} 
Matching \\
NR
\end{tabular}} & \multirow{3}{*}{\begin{tabular}{|l}
$\begin{array}{l}\text { Mean age } \\
\text { (years) }\end{array}$ \\
NR
\end{tabular}} & \multirow{3}{*}{\begin{tabular}{|l} 
Mean BMI \\
NR
\end{tabular}} & \multirow{3}{*}{\begin{tabular}{|l|}
$\begin{array}{l}\text { Method of } \\
\text { assessment }\end{array}$ \\
$\begin{array}{l}\text { Inductively } \\
\text { coupled plasma } \\
\text { spectrometer }\end{array}$ \\
\end{tabular}} & \multirow{3}{*}{\begin{tabular}{|l|}
$\begin{array}{l}\text { Magnesium } \\
\text { concentration, } \\
\text { mmol/L } \\
(\text { mean } \pm \text { SD })\end{array}$ \\
Cases: \\
$0.88 \pm 0.28$ \\
Controls: \\
$1.45 \pm 0.27$ \\
\end{tabular}} & \multirow{3}{*}{$\begin{array}{l}\text { NO } \\
7\end{array}$} \\
\hline \multirow[b]{2}{*}{$\begin{array}{l}\text { Zhou (1) (2019; } \\
\text { China) }\end{array}$} & \multirow[b]{2}{*}{ CC } & \multirow[b]{2}{*}{ Serum } & & Cases: 12 & & & & & & \\
\hline & & & $\begin{array}{l}\text { Prediabetes } \\
\text { (IGF)/healthy }\end{array}$ & Controls: 50 & & & & & & \\
\hline \multirow{2}{*}{$\begin{array}{l}\text { Zhou (2) (2019; } \\
\text { China) }\end{array}$} & \multirow[b]{2}{*}{ CC } & \multirow[b]{2}{*}{ Serum } & \multirow{2}{*}{$\begin{array}{l}\text { Prediabetes } \\
\text { (IGT)/healthy }\end{array}$} & \begin{tabular}{|l} 
Cases: 15 \\
\end{tabular} & \multirow[b]{2}{*}{ NR } & \multirow[b]{2}{*}{ NR } & \multirow[b]{2}{*}{ NR } & \multirow{2}{*}{\begin{tabular}{|l} 
Inductively \\
coupled plasma \\
spectrometer
\end{tabular}} & \multirow{2}{*}{\begin{tabular}{|l|} 
Cases: \\
$0.94 \pm 0.21$ \\
Controls: \\
$1.45 \pm 0.27$ \\
\end{tabular}} & \multirow[b]{2}{*}{7} \\
\hline & & & & Controls: 50 & & & & & & \\
\hline \multirow[b]{2}{*}{$\begin{array}{l}\text { Rahim (2018; } \\
\text { Bangladesh) }\end{array}$} & \multirow[b]{2}{*}{ CC } & \multirow[b]{2}{*}{ Serum } & \multirow[b]{2}{*}{$\begin{array}{l}\text { Prediabetes/ } \\
\text { healthy }\end{array}$} & Cases: 50 & \multirow[b]{2}{*}{ Age, Sex } & \multirow[b]{2}{*}{\begin{tabular}{|l} 
Case: 43.68 \\
Control: 43.26
\end{tabular}} & \multirow[b]{2}{*}{\begin{tabular}{|l} 
Case: 27.70 \\
Control: 25.33
\end{tabular}} & \multirow[b]{2}{*}{ NR } & \multirow{2}{*}{\begin{tabular}{|l|} 
Cases: \\
$0.70 \pm 0.14$ \\
Controls: \\
$0.85 \pm 0.15$
\end{tabular}} & \multirow[b]{2}{*}{9} \\
\hline & & & & Controls: 50 & & & & & & \\
\hline \multirow[b]{2}{*}{$\begin{array}{l}\text { Chen (2017; } \\
\text { China) }\end{array}$} & & & & Prediabetes: 867 & & & & Inductively & Cases: & \\
\hline & $\mathrm{CC}$ & Plasma & $\begin{array}{l}\text { Prediabetes/ } \\
\text { healthy }\end{array}$ & Healthy: 2105 & Age, Sex & \begin{tabular}{|l|} 
Case: 52.96 \\
Control: 52.21
\end{tabular} & $\begin{array}{l}\text { Case: } 25.09 \\
\text { Control: } 23.30\end{array}$ & $\begin{array}{l}\text { coupled plasma } \\
\text { mass spectrom- } \\
\text { etry }\end{array}$ & \begin{tabular}{|l}
$0.88 \pm 0.12$ \\
Controls: \\
$0.91 \pm 0.11$
\end{tabular} & 8 \\
\hline & & & & Prediabetes: 145 & & & & Flame atomic & Prediabetes: & \\
\hline $\begin{array}{l}\text { Fang (2016; } \\
\text { Chin) }\end{array}$ & $\mathrm{NC}$ & Serum & $\begin{array}{l}\text { Prediabetes/ } \\
\text { healthy }\end{array}$ & Healthy: 145 & Gender, Age & \begin{tabular}{|l|} 
Case: 60.23 \\
Control: 60.19
\end{tabular} & $\begin{array}{l}\text { Case: } 24.05 \\
\text { Control: } 23.23\end{array}$ & $\begin{array}{l}\text { absorption } \\
\text { spectroscopy }\end{array}$ & $\begin{array}{l}0.90 \pm 3.13 \\
\text { Healthy: } \\
0.97 \pm 3.73\end{array}$ & 9 \\
\hline & & & & \begin{tabular}{|l|} 
Prediabetes: 224 \\
\end{tabular} & & & & & Prediabetes: & \\
\hline $\begin{array}{l}\text { Spiga (2019; } \\
\text { Italy) }\end{array}$ & CS & Serum & $\begin{array}{l}\text { Prediabetes/ } \\
\text { healthy }\end{array}$ & Healthy: 365 & NR & $\begin{array}{l}\text { Prediabetes: } 51 \\
\text { Healthy: } 44\end{array}$ & $\begin{array}{l}\text { Prediabetes: } 31.5 \\
\text { Healthy: } 30.1\end{array}$ & $\begin{array}{l}\text { Colorimetric } \\
\text { method assay }\end{array}$ & $\begin{array}{l}0.81 \pm 0.07 \\
\text { Healthy: } \\
0.82 \pm 0.06\end{array}$ & 5 \\
\hline & & & & \begin{tabular}{|l|} 
Prediabetes: 85 \\
\end{tabular} & & & & & & \\
\hline $\begin{array}{l}\text { Aksit (2019; } \\
\text { Turkey) }\end{array}$ & CS & Serum & $\begin{array}{l}\text { Prediabetes/ } \\
\text { healthy }\end{array}$ & Healthy: 137 & NR & $\begin{array}{l}\text { Prediabetes: } 34.5 \\
\text { Healthy: } 29.75\end{array}$ & NR & $\begin{array}{l}\text { Photometric } \\
\text { method }\end{array}$ & $\begin{array}{l}0.84 \pm 0.03 \\
\text { Healthy: } \\
0.86 \pm 0.05\end{array}$ & 5 \\
\hline & & & & \begin{tabular}{|l|} 
Prediabetes: \\
1346
\end{tabular} & & & & $\begin{array}{l}\text { Colorimet- } \\
\text { ric endpoint } \\
\text { method and the }\end{array}$ & Prediabetes: & \\
\hline $\begin{array}{l}\text { Netherlands) } \\
\text { Neom (201/; }\end{array}$ & CS & Serum & $\begin{array}{l}\text { Prediabetes/ } \\
\text { healthy }\end{array}$ & Healthy: 7209 & NR & $\begin{array}{l}\text { Prediabetes: } 66.6 \\
\text { Healthy: } 64.3\end{array}$ & $\begin{array}{l}\text { Prediabetes: } 28.5 \\
\text { Healthy: } 26.7\end{array}$ & $\begin{array}{l}\text { method and the } \\
\text { Roche/Hitachi } \\
\text { Cobas c501 } \\
\text { Analyzer }\end{array}$ & $\begin{array}{l}0.84 \pm 0.06 \\
\text { Healthy: } \\
0.85 \pm 0.06\end{array}$ & 6 \\
\hline & & & & Prediabetes: 35 & & & Prediabetes: & & Prediabetes: & \\
\hline $\begin{array}{l}\text { Yadav (2017; } \\
\text { india) }\end{array}$ & CS & Serum & $\begin{array}{l}\text { Prediabetes/ } \\
\text { healthy }\end{array}$ & Healthy: 35 & Age & $\begin{array}{l}\text { Prediabetes: } 36.8 \\
\text { Healthy: } 34.8\end{array}$ & $\begin{array}{l}25.35 \\
\text { Healthy: } 22.52\end{array}$ & $\begin{array}{l}\text { Semi-automated } \\
\text { analyser }\end{array}$ & $\begin{array}{l}0.56 \pm 0.15 \\
\text { Healthy: } \\
0.87 \pm 0.09\end{array}$ & 7 \\
\hline & & & Prediabetes & Prediabetes: 78 & & & & & Prediabetes: & \\
\hline $\begin{array}{l}\text { Chambers (1) } \\
\text { (2006; USA) }\end{array}$ & CS & Serum & $\begin{array}{l}\text { (African Ameri- } \\
\text { can)/ healthy }\end{array}$ & Healthy: 109 & NR & NR & NR & NR & $\begin{array}{l}0.85 \pm 0.08 \\
\text { Healthy: } \\
0.85 \pm 0.08\end{array}$ & 5 \\
\hline & & & Prediabetes & \begin{tabular}{|l|} 
Prediabetes: 70 \\
\end{tabular} & & & & & Prediabetes: & \\
\hline $\begin{array}{l}\text { Chambers (2) } \\
\text { (2006; USA) }\end{array}$ & CS & Serum & $\begin{array}{l}\text { (Hispanic)/ } \\
\text { healthy }\end{array}$ & Healthy: 169 & NR & NR & NR & NR & $\begin{array}{l}0.83 \pm 0.08 \\
\text { Healthy: } \\
0.83 \pm 0.09\end{array}$ & 5 \\
\hline & & & & Prediabetes: 52 & & & & & Prediabetes: & \\
\hline $\begin{array}{l}\text { Lind (1990; } \\
\text { Sweden) }\end{array}$ & CS & Serum & $\begin{array}{l}\text { Prediabetes/ } \\
\text { healthy }\end{array}$ & Healthy: 52 & Age, Sex & NR & NR & $\begin{array}{l}\text { Atomic absorp- } \\
\text { tion }\end{array}$ & $\begin{array}{l}0.79 \pm 0.06 \\
\text { Healthy: } \\
0.85 \pm 0.06\end{array}$ & 6 \\
\hline
\end{tabular}

Table 1. Characteristics of included studies. BMI Body Mass Index, CC Case-Control, CS cross sectional, NC Nested case-control, $M g$ Magnesium, ADA American Diabetes Association, WHO World Health Organization, NR Not reported.

Overall meta-analysis. Forest plot showing the association between magnesium level and prediabetes depicted in Fig. 2. The random effects meta-analysis indicated that subjects with prediabetes had a significantly lower serum magnesium concentration compared with their healthy controls (WMD $=-0.07 \mathrm{mmol} / \mathrm{L} ; 95 \%$ CI: $-0.09,-0.05 \mathrm{mmol} / \mathrm{L}, \mathrm{P}<0.001$, Fig. 2). However, evaluation of studies in terms of heterogeneity demonstrated a high degree of heterogeneity $\left(\mathrm{I}^{2}=95.6 \%, \mathrm{P}<0.001\right)$. Following the subgroup analysis based on sample type (serum and plasma), publication year $(\leq 2010$ and $>2010)$ and study design (case-control and cross-sectional), we could not find the origin of heterogeneity (Table 3). The subgroup analysis showed that magnesium concentrations in prediabetic patients were significantly lower than those in healthy individuals in studies published before $2010(\mathrm{WMD}=-0.12 \mathrm{mmol} / \mathrm{L}, 95 \% \mathrm{CI}-0.14,-0.09)$. However, there was no significant difference in magnesium concentration when compared between healthy controls and prediabetes subjects in studies published 2010 onwards $(\mathrm{WMD}=-0.02 \mathrm{mmol} / \mathrm{L}, 95 \% \mathrm{CI}-0.06,0.02)$. The results of subgroup analysis based on the quality of the study also indicated that compared to healthy individuals, the concentration of magnesium in prediabetic individuals is lower in high quality studies (WMD $=-0.87 \mathrm{mmol} / \mathrm{L}, 95 \% \mathrm{CI}-1.29,-0.45$ ) than in moderate quality studies (WMD $=-0.37 \mathrm{mmol} / \mathrm{L}, 95 \% \mathrm{CI}-0.61,-0.13)$. Moreover, the analysis showed that magnesium concentrations in the pre-diabetic group were lower than in healthy subjects in European studies 


\begin{tabular}{|c|c|c|c|c|c|c|c|c|c|}
\hline \multicolumn{10}{|c|}{ Case-control studies } \\
\hline Publications & $\begin{array}{l}\text { Case definition } \\
\text { adequate }\end{array}$ & $\begin{array}{l}\text { Representativeness } \\
\text { of the cases }\end{array}$ & $\begin{array}{l}\text { Selection of } \\
\text { controls }\end{array}$ & $\begin{array}{l}\text { Definition of } \\
\text { controls }\end{array}$ & $\begin{array}{l}\begin{array}{l}\text { Comparability } \\
\text { of cases and } \\
\text { controls }\end{array} \\
\end{array}$ & $\begin{array}{l}\text { Ascertainment } \\
\text { of exposure }\end{array}$ & \begin{tabular}{|l|}
$\begin{array}{l}\text { Same } \\
\text { method of } \\
\text { ascertainment }\end{array}$ \\
\end{tabular} & \begin{tabular}{|l}
$\begin{array}{l}\text { Non-response } \\
\text { rate }\end{array}$ \\
\end{tabular} & NOS \\
\hline $\begin{array}{l}\text { Chen et al. } \\
\text { (2017) }\end{array}$ & * & * & - & * & ** & * & * & * & 8 \\
\hline $\begin{array}{l}\text { Fang et al. } \\
(2016)\end{array}$ & * & * & * & * & $* *$ & * & * & * & 9 \\
\hline $\begin{array}{l}\text { Rahim et al. } \\
(2018)\end{array}$ & * & * & * & * & $* *$ & * & * & * & 9 \\
\hline $\begin{array}{l}\text { Zhou et al. } \\
\text { (2019) }\end{array}$ & * & * & * & * & - & * & * & * & 7 \\
\hline \multicolumn{10}{|c|}{ Cross sectional studies } \\
\hline Publications & \begin{tabular}{|l|} 
Representativeness \\
of the sample
\end{tabular} & Sample size & $\begin{array}{l}\text { Non- } \\
\text { respondents }\end{array}$ & $\begin{array}{l}\text { Ascertainment } \\
\text { of the exposure }\end{array}$ & Comparability & $\begin{array}{l}\text { Ascertainment } \\
\text { of outcome }\end{array}$ & Statistical test & & NOS \\
\hline $\begin{array}{l}\text { Kieboom et al. } \\
(2017)\end{array}$ & * & - & * & * & - & $* *$ & * & & 6 \\
\hline $\begin{array}{l}\text { Lind et al. } \\
\text { (1990) }\end{array}$ & * & - & * & * & ** & - & * & & 6 \\
\hline $\begin{array}{l}\text { Yadav et al. } \\
(2017)\end{array}$ & * & - & * & * & * & $* *$ & * & & 7 \\
\hline $\begin{array}{l}\text { Spiga et al. } \\
\text { (2019) }\end{array}$ & * & - & * & * & - & * & * & & 5 \\
\hline $\begin{array}{l}\text { Chambers et al. } \\
\text { (2006) }\end{array}$ & * & - & * & * & - & * & * & & 5 \\
\hline $\begin{array}{l}\text { Aksit et al. } \\
\text { (2019) }\end{array}$ & * & - & * & * & - & ** & * & & 5 \\
\hline
\end{tabular}

Table 2. Quality of included studies according to Newcastle-Ottawa Scale (NOS).

Study

ID
WMD $(95 \%$ CI) Weight

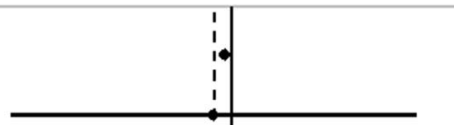

$-0.03(-0.04,-0.02)$

12.19

Chen (2017)

Fang (2016)

Kieboom (2017)

Lind (1990)

Rahim (2018)

Zhou(1) (2019)

Zhou(2) (2019)

Yadav (2017)

Spiga (2019)

Chambers(1) (2006)

Chambers(2) (2006)

Aksit (2019)

Overall $(\mathrm{I}$-squared $=95.6 \%, \mathrm{p}=0.000)$

NOTE: Weights are from random effects analysis
$-0.07(-0.86,0.72) \quad 0.08$

$-0.01(-0.01,-0.01) \quad 12.41$

$-0.06(-0.08,-0.04) \quad 11.05$

$-0.15(-0.21,-0.09) \quad 7.05$

$-0.57(-0.75,-0.39) \quad 1.50$

$-0.51(-0.64,-0.38) \quad 2.49$

$-0.31(-0.37,-0.25) \quad 6.93$

$-0.01(-0.02,0.00) \quad 12.09$

$0.00(-0.02,0.02) \quad 11.03$

$0.00(-0.02,0.02) \quad 11.04$

$-0.02(-0.03,-0.01) \quad 12.12$

$-0.07(-0.09,-0.05) \quad 100.00$

Figure 2. Forest plot for the association between magnesium level and prediabetes expressed as mean difference between case and control groups. The area of each square is proportional to the inverse of the variance of the WMD. Horizontal lines represent 95\%Cis. Diamonds represent pooled estimates from random-effects analysis. WMD, weighted mean difference. 


\begin{tabular}{|c|c|c|c|c|c|c|}
\hline Sub grouped by & No & WMD $(95 \%$ CI $)$ & P-value & P-Heterogeneity & $I^{2}(\%)$ & $\begin{array}{l}\text { P- between subgroup } \\
\text { heterogeneity }\end{array}$ \\
\hline \multicolumn{6}{|l|}{ Publication year } & \multirow{3}{*}{0.362} \\
\hline$\leq 2010$ & 2 & $-0.02(-0.06,0.02)$ & 0.318 & $<0.001$ & $88.4 \%$ & \\
\hline$>2010$ & 8 & $-0.12(-0.14,-0.09)$ & $<0.001$ & $<0.001$ & $97.8 \%$ & \\
\hline \multicolumn{7}{|l|}{ Sample } \\
\hline Serum & 9 & $-0.10(-0.12,-0.07)$ & $<0.001$ & $<0.001$ & $97.3 \%$ & \multirow{2}{*}{$<0.001$} \\
\hline Plasma & 1 & $-0.03(-0.04,-0.02)$ & $<0.001$ & - & $0 \%$ & \\
\hline \multicolumn{7}{|l|}{ Study design } \\
\hline Case-control & 3 & $-0.25(-0.45,-0.05)$ & 0.016 & $<0.001$ & $98.6 \%$ & \multirow{3}{*}{$<0.001$} \\
\hline Cross- sectional & 6 & $-0.09(-0.11,-0.06)$ & $<0.001$ & $<0.001$ & $95.1 \%$ & \\
\hline Nested case-control & 1 & $-0.07(-0.86,0.72)$ & 0.863 & - & $0 \%$ & \\
\hline \multicolumn{7}{|l|}{ Region } \\
\hline US & 2 & $0.00(-0.20,0.20)$ & 1.00 & 1.00 & $0 \%$ & \multirow{3}{*}{$<0.001$} \\
\hline European & 2 & $-0.35(-0.62,0.07)$ & 0.015 & $<0.001$ & $87.3 \%$ & \\
\hline Others & 6 & $-1.09(-1.57,-0.61)$ & $<0.001$ & $<0.001$ & $94.7 \%$ & \\
\hline \multicolumn{7}{|l|}{ Quality score } \\
\hline High & 7 & $-0.87(-1.29,-0.45)$ & $<0.001$ & $<0.001$ & $94.2 \%$ & \multirow{2}{*}{0.02} \\
\hline Moderate & 3 & $-0.37(-.0 .61,-0.13)$ & 0.002 & $<0.001$ & $84.7 \%$ & \\
\hline \multicolumn{7}{|l|}{ Comparability } \\
\hline Complete adjustment & 4 & $-0.53(-0.91,-0.15)$ & 0.006 & $<0.001$ & $89.8 \%$ & \multirow{2}{*}{0.05} \\
\hline No or incomplete adjustment & 6 & $-0.74(-1.08,-0.40)$ & $<0.001$ & $<0.001$ & $93.8 \%$ & \\
\hline
\end{tabular}

Table 3. Subgroup analysis to assess the magnesium concentrations in subjects with prediabetes. WMD weighted mean difference.

$(\mathrm{WMD}=-0.35 \mathrm{mmol} / \mathrm{L}, 95 \% \mathrm{CI}-0.62,-0.07)$, while in US studies, magnesium concentrations were not significantly different between the healthy and pre-diabetic groups (WMD $=-0.00 \mathrm{mmol} / \mathrm{L}, 95 \% \mathrm{CI}-0.20,0.20$ ). Our analysis also highlighted that studies were performed in another region reported the lowest magnesium concentration with prediabetes compared to their healthy counterparts $(\mathrm{WMD}=-1.09 \mathrm{mmol} / \mathrm{L}, 95 \% \mathrm{CI}-1.57,-0.61)$. Subgroup analysis of our study based on comparability also showed that magnesium concentrations in prediabetics in studies that have no or incomplete adjustment (WMD $=-0.74 \mathrm{mmol} / \mathrm{L}, 95 \% \mathrm{CI}-1.08,-0.40$ ) were lower than studies had complete adjustment $(\mathrm{WMD}=-0.53 \mathrm{mmol} / \mathrm{L}, 95 \% \mathrm{CI}-0.91,-0.15)$. Sensitivity analysis showed that the omission of each individual study had no effect on the overall results.

Publication bias. Although a comprehensive search was performed to reduce the possibility of publication bias, we also use Begg's and Egger's test to detect any potential publication bias. Begg's test did not find significant publication bias $(\mathrm{P}=0.1)$ (Fig. 3$)$, but Egger's test indicated significant publication bias $(\mathrm{P}=0.008)$. Finally, trimand-fill analysis indicated no trimming and data unchanged.

\section{Discussion}

The role of magnesium in the management of chronic diseases such as metabolic syndrome, cardiovascular disease, cerebrovascular accident, hypertension and type 2 diabetes mellitus has received a great attention in recent decades $^{40-45}$. Hypomagnesemia is one of the causes of insulin resistance, high blood glucose, and cardiovascular complications of diabetes ${ }^{46,47}$. Patients with diabetes had increased magnesium excretion in the urine, which is due to hyperglycemia, hyperfiltration, and the effect of insulin on the renal channels of magnesium ${ }^{48}$. Although among people with prediabetes serum glucose levels are below the threshold for magnesium extraction in the urine and they are unlikely to affect serum magnesium levels ${ }^{37}$, but a number of studies have shown that magnesium levels in subjects with prediabetes are also lower than healthy individuals ${ }^{19,22,34}$. Previous meta-analysis has reported an inverse relationship between magnesium levels and magnesium intake with the risk of diabetes, and also confirmed the beneficial effect of magnesium supplementation in the management of glucose disorders ${ }^{8,9,16,49}$.

Although many studies have examined the association between magnesium and diabetes, there are limited studies on the association of magnesium with prediabetes and its progression to diabetes. To the best of our knowledge, this is the first systematic review and meta-analysis to examine the association between serum magnesium levels and prediabetes. The present meta-analysis of ten observational studies involving 2979 cases and 10,476 controls detected that serum magnesium levels are lower in prediabetes patients compared with their healthy controls. Significant heterogeneity was observed among the included studies. Following the subgroup analysis based on sample type, publication year and study design, we could not identify the source of heterogeneity, which may be attributed to other study parameters such as the magnesium assessment methods, criteria for diagnosing prediabetes, and ethnic populations. Due to lack of sufficient information about them, we could not perform subgroup analysis and find the source of heterogeneity.

Magnesium plays an important role in the activity of more than 300 enzymes, including all enzymes that use or synthesize adenosine triphosphate (ATP), as well as enzymes effective in glucose metabolism ${ }^{50,51}$. Various 


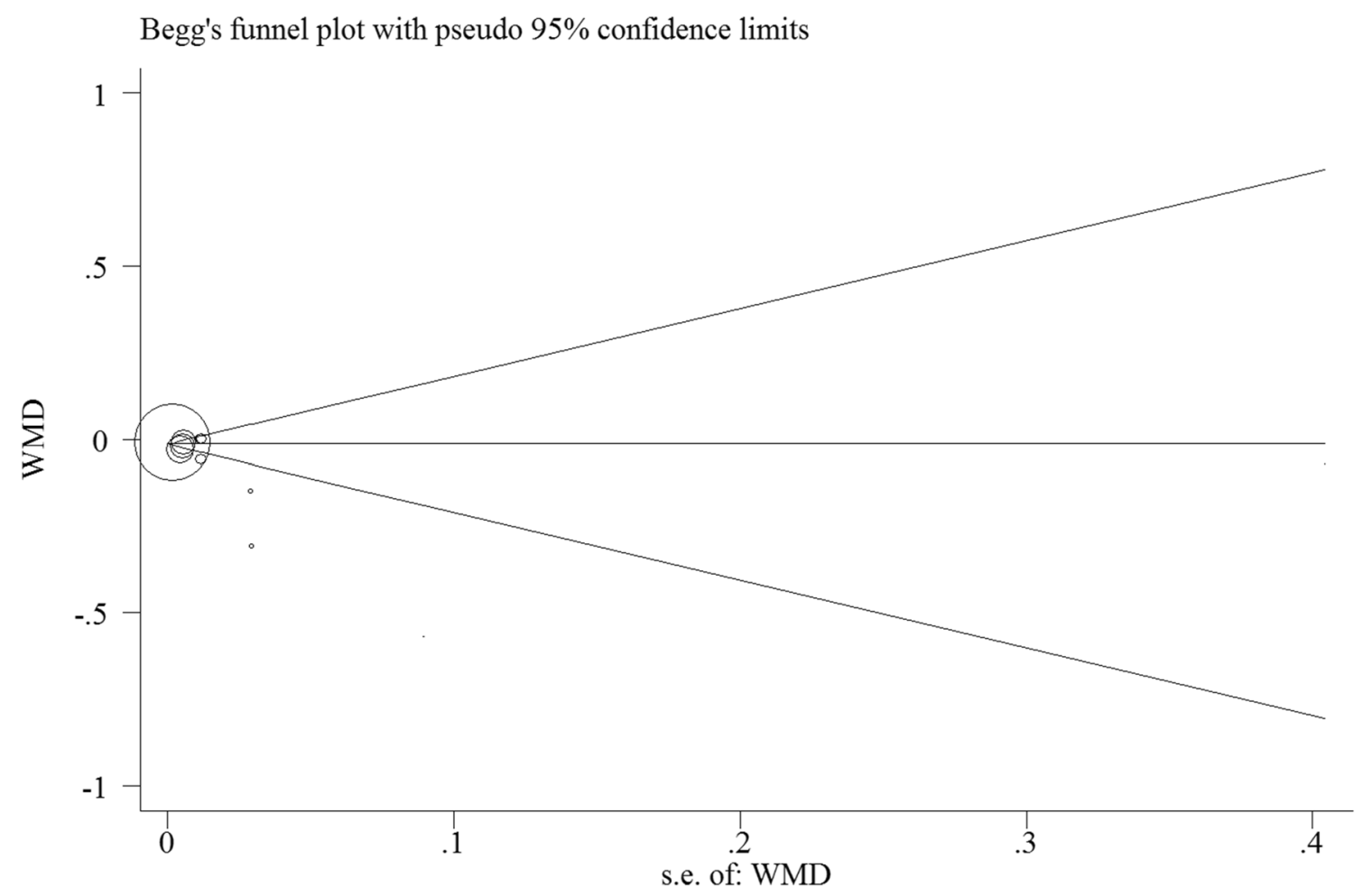

Figure 3. Funnel plot of the weighted mean difference (WMD) versus the s.e. of the weighted mean difference (WMD). All statistical analyses were performed using Stata version 14 (StataCorp. 2015. Stata Statistical Software: Release 14. College Station, TX: StataCorp LP, www.stata.com).

studies have confirmed the fundamental role of magnesium in glucose metabolism, including cellular uptake of glucose, glucose excretion, insulin secretion and insulin function ${ }^{52-55}$. Among patients with impaired glucose metabolism, there is a higher renal magnesium wasting due to reduced tubular magnesium reabsorption resulting from glucose-induced osmotic diuresis ${ }^{20,48,56}$. Low levels of magnesium can inhibit phosphorus-dependent reactions and the activity of many enzymes involved in glucose metabolism, thereby reducing insulin secretion, preventing cellular glucose uptake and promoting the development of metabolic glucose disorders ${ }^{50,57}$. Magnesium deficiency can cause serious complications in glucose disorders, such as cardiac arrhythmia, hypertension and myocardial infarction, thus, considering magnesium status can be important in the management of prediabetes and its progression to diabetes ${ }^{58,59}$.

Several limitations of our meta-analysis should be acknowledged. Firstly, the design of all studies included in the current meta-analysis are observational, so we could not infer a causal association between serum magnesium level and prediabetes. Secondly, most of included studies did not make adjustment for the potential confounders, especially age and gender. Thus, the residual confounder may affect the results. Thirdly, the diagnostic criteria for prediabetes were different in the studies that could affect the outcome. Fourthly, we use the "mean difference" of serum magnesium levels as an effect size which are not the best effect measure compared to odds ratio or hazard ratio with $95 \%$ CI to evaluate the relationship between outcome and exposure. Fifthly, the included studies did not measure the magnesium intake which could be attributed to the serum magnesium levels. In addition, we have excluded studies published in a non-English language that may affect the final outcome. Finally, we observed a significant heterogeneity among included studies. However, our attempts to detect the potential source of heterogeneity through different subgroup analysis were unsuccessful. Therefore, the results should be interpreted with caution.

\section{Conclusions}

The present meta-analysis indicated that circulating magnesium levels in people with prediabetes were significantly lower than healthy controls, confirming that magnesium deficiency may play a role in development of prediabetes. However, it should be noted that due to the small estimate effect, the results should be interpreted with caution. Assessment of magnesium levels in subjects with prediabetes and improvement of possible deficiencies may prevent its progression to diabetes. Further prospective cohort studies with larger sample size and robust design are warranted to confirm present findings.

Received: 7 June 2021; Accepted: 13 December 2021

Published online: 22 December 2021 


\section{References}

1. DeFronzo, R. A. \& Abdul-Ghani, M. Assessment and treatment of cardiovascular risk in prediabetes: Impaired glucose tolerance and impaired fasting glucose. Am. J. Cardiol. 108, 3B-24B (2011).

2. Association, A. D. Diagnosis and classification of diabetes mellitus. Diabetes Care 33, S62-S69 (2010).

3. World Health Organization. Definition, Diagnosis and Classification of Diabetes Mellitus and its Complications: Report of a WHO Consultation. Part 1, Diagnosis and Classification of Diabetes Mellitus. (World Health Organization, 1999).

4. Gavin, J. R. III., Alberti, K., Davidson, M. B. \& DeFronzo, R. A. Report of the expert committee on the diagnosis and classification of diabetes mellitus. Diabetes Care 20, 1183 (1997).

5. Yudkin, J. S. \& Montori, V. M. The epidemic of pre-diabetes: The medicine and the politics. BMJ 349, 4485 (2014).

6. Liu, J. et al. Ten-year risk of cardiovascular incidence related to diabetes, prediabetes, and the metabolic syndrome. Am. Heart J. 153, 552-558 (2007).

7. Liamis, G., Liberopoulos, E., Barkas, F. \& Elisaf, M. Diabetes mellitus and electrolyte disorders. World J. Clin. Cases 2, 488 (2014).

8. Dong, J.-Y., Xun, P., He, K. \& Qin, L.-Q. Magnesium intake and risk of type 2 diabetes: Meta-analysis of prospective cohort studies. Diabetes Care 34, 2116-2122 (2011).

9. Wu, J., Xun, P., Tang, Q., Cai, W. \& He, K. Circulating magnesium levels and incidence of coronary heart diseases, hypertension, and type 2 diabetes mellitus: A meta-analysis of prospective cohort studies. Nutr. J. 16, 1-13 (2017).

10. Kao, W. L. et al. Serum and dietary magnesium and the risk for type 2 diabetes mellitus: The atherosclerosis risk in communities study. Arch. Intern. Med. 159, 2151-2159 (1999).

11. Gröber, U., Schmidt, J. \& Kisters, K. Magnesium in prevention and therapy. Nutrients 7, 8199-8226 (2015).

12. Bertinato, J. et al. Lower serum magnesium concentration is associated with diabetes, insulin resistance, and obesity in South Asian and white Canadian women but not men. Food Nutr. Res. 59, 25974 (2015).

13. Chakraborti, S. et al. Protective role of magnesium in cardiovascular diseases: A review. Mol. Cell. Biochem. 238, 163-179 (2002).

14. Dyckner, T. \& Wester, P. Effect of magnesium on blood pressure. Br. Med. J. 286, 1847-1849 (1983).

15. Fontana, L. et al. Decreased consumption of branched-chain amino acids improves metabolic health. Cell Rep. 16, 520-530. https:// doi.org/10.1016/j.celrep.2016.05.092 (2016).

16. Veronese, N. et al. Effect of magnesium supplementation on glucose metabolism in people with or at risk of diabetes: A systematic review and meta-analysis of double-blind randomized controlled trials. Eur. J. Clin. Nutr. 70, 1354-1359 (2016).

17. Simental-Mendia, L. E., Sahebkar, A., Rodriguez-Moran, M. \& Guerrero-Romero, F. A systematic review and meta-analysis of randomized controlled trials on the effects of magnesium supplementation on insulin sensitivity and glucose control. Pharmacol. Res. 111, 272-282 (2016).

18. Zhou, Q., Guo, W., Jia, Y. \& Xu, J. Serum and urinary selenium status in patients with the pre-diabetes and diabetes in Northeast China. Biol. Trace Elem. Res. 191, 61-69 (2019).

19. Chen, S. et al. Association of plasma magnesium with prediabetes and type 2 diabetes mellitus in adults. Sci. Rep. 7, 1-8 (2017).

20. Lind, L., Lithell, H., Hvarfner, A. \& Ljunghall, S. Indices of mineral metabolism in subjects with an impaired glucose tolerance. Exp. Clin. Endocrinol. Diabetes 96, 109-112 (1990).

21. Chambers, E. C. et al. Serum magnesium and type-2 diabetes in African Americans and Hispanics: A New York cohort. J. Am. Coll. Nutr. 25, 509-513 (2006).

22. Rahim, M. A. \& Mitra, P. Serum magnesium level of newly detected patients with glucose intolerance and its comparison with serum magnesium level of age and sex matched healthy volunteers. J. Med. 19, 95-99 (2018).

23. Moher, D., Liberati, A., Tetzlaff, J., Altman, D. G. \& Group, P. Preferred reporting items for systematic reviews and meta-analyses: The PRISMA statement. PLoS Med. 6, e1000097 (2009).

24. Larsson, S. \& Wolk, A. Magnesium intake and risk of type 2 diabetes: A meta-analysis. J. Intern. Med. 262, 208-214 (2007).

25. Dibaba, D. T. et al. The effect of magnesium supplementation on blood pressure in individuals with insulin resistance, prediabetes, or noncommunicable chronic diseases: A meta-analysis of randomized controlled trials. Am. J. Clin. Nutr. 106, 921-929 (2017).

26. Stang, A. Critical evaluation of the Newcastle-Ottawa scale for the assessment of the quality of nonrandomized studies in metaanalyses. Eur. J. Epidemiol. 25, 603-605 (2010).

27. Herzog, R. et al. Are healthcare workers' intentions to vaccinate related to their knowledge, beliefs and attitudes? A systematic review. BMC Public Health 13, 1-17 (2013).

28. Hozo, S. P., Djulbegovic, B. \& Hozo, I. Estimating the mean and variance from the median, range, and the size of a sample. BMC Med. Res. Methodol. 5, 1-10 (2005).

29. DerSimonian, R. \& Laird, N. Meta-analysis in clinical trials. Control. Clin. Trials 7, 177-188 (1986).

30. Higgins, J. P. \& Thompson, S. G. Quantifying heterogeneity in a meta-analysis. Stat. Med. 21, 1539-1558 (2002).

31. Egger, M., Smith, G. D., Schneider, M. \& Minder, C. Bias in meta-analysis detected by a simple, graphical test. BMJ 315, 629-634 (1997).

32. Duval, S. The trim and fill method. Publication bias in meta-analysis: Prevention, assessment and adjustments, 127-144 (2005).

33. Li, Y. et al. Magnesium status and dietary intake of mid-old people in a rural area of China. Magnes. Res. 22, 66-71 (2009).

34. Xu, J. et al. Associations of serum and urinary magnesium with the pre-diabetes, diabetes and diabetic complications in the Chinese Northeast population. PLOS ONE 8, e56750 (2013).

35. Aksit, M., Aksit, M. Z. \& Basok, B. I. The impact of magnesium on glycemic regulation. Int. J. Med. Biochem. 2, 13-18 (2019).

36. Fang, C. et al. Association of serum magnesium level with odds of prediabetes and diabetes in a southern Chinese population: A prospective nested case-control study. Biol. Trace Elem. Res. 172, 307-314 (2016).

37. Kieboom, B. C. et al. Serum magnesium and the risk of prediabetes: A population-based cohort study. Diabetologia $\mathbf{6 0}, 843-853$ (2017).

38. Spiga, R. et al. Are circulating Mg2+ levels associated with glucose tolerance profiles and incident type 2 diabetes?. Nutrients $\mathbf{1 1}$, 2460 (2019).

39. Yadav, C. et al. Association of serum selenium, zinc and magnesium levels with glycaemic indices and insulin resistance in prediabetes: A cross-sectional study from South India. Biol. Trace Elem. Res. 175, 65-71 (2017).

40. Champagne, C. M. Magnesium in hypertension, cardiovascular disease, metabolic syndrome, and other conditions: A review. Nutr. Clin. Pract. 23, 142-151 (2008).

41. Sarrafzadegan, N., Khosravi-Boroujeni, H., Lotfizadeh, M., Pourmogaddas, A. \& Salehi-Abargouei, A. Magnesium status and the metabolic syndrome: A systematic review and meta-analysis. Nutrition 32, 409-417 (2016).

42. Ghayyur, A., Hussain, S. S. \& Butt, A. Risk factors of hypomagnesemia in patients with acute ischemic stroke (AIS): A cross sectional study of a tertiary care hospital, Lahore Pakistan during 2015. FUUAST J. Biol. 7, 23-32 (2017).

43. Kostov, K. \& Halacheva, L. Role of magnesium deficiency in promoting atherosclerosis, endothelial dysfunction, and arterial stiffening as risk factors for hypertension. Int. J. Mol. Sci. 19, 1724 (2018).

44. Shi, Z. \& Abou-Samra, A. B. Association of low serum magnesium with diabetes and hypertension: Findings from Qatar Biobank Study. Diabetes Res. Clin. Pract. 158, 107903 (2019).

45. Kumar, P., Bhargava, S., Agarwal, P. K., Garg, A. \& Khosla, A. Association of serum magnesium with type 2 diabetes mellitus and diabetic retinopathy. J. Fam. Med. Primary Care 8, 1671 (2019). 
46. Rodríguez-Hernández, H., Gonzalez, J. L., Rodríguez-Morán, M. \& Guerrero-Romero, F. Hypomagnesemia, insulin resistance, and non-alcoholic steatohepatitis in obese subjects. Arch. Med. Res. 36, 362-366 (2005).

47. Dasgupta, A., Sarma, D. \& Saikia, U. K. Hypomagnesemia in type 2 diabetes mellitus. Indian J. Endocrinol. Metab. 16, 1000 (2012).

48. Gommers, L. M., Hoenderop, J. G., Bindels, R. J. \& de Baaij, J. H. Hypomagnesemia in type 2 diabetes: A vicious circle?. Diabetes 65, 3-13 (2016).

49. Song, Y., He, K., Levitan, E., Manson, J. \& Liu, S. Effects of oral magnesium supplementation on glycaemic control in type 2 diabetes: A meta-analysis of randomized double-blind controlled trials. Diabet. Med. 23, 1050-1056 (2006).

50. Paolisso, G., Scheen, A., d’Onofrio, F. \& Lefebvre, P. Magnesium and glucose homeostasis. Diabetologia 33, 511-514 (1990).

51. Yajnik, C., Smith, R., Hockaday, T. \& Ward, N. Fasting plasma magnesium concentrations and glucose disposal in diabetes. Br. Med. J. 288, 1032-1034 (1984).

52. Ma, J. et al. Associations of serum and dietary magnesium with cardiovascular disease, hypertension, diabetes, insulin, and carotid arterial wall thickness: The ARIC study. J. Clin. Epidemiol. 48, 927-940 (1995).

53. Barbagallo, M. et al. Role of magnesium in insulin action, diabetes and cardio-metabolic syndrome X. Mol. Aspects Med. 24, 39-52 (2003).

54. Takaya, J., Higashino, H. \& Kobayashi, Y. Intracellular magnesium and insulin resistance. Magnes. Res. 17, 126-136 (2004).

55. Kandeel, F. R., Balon, E., Scott, S. \& Nadler, J. L. Magnesium deficiency and glucose metabolism in rat adipocytes. Metabolism 45, 838-843 (1996).

56. Tosiello, L. Hypomagnesemia and diabetes mellitus: A review of clinical implications. Arch. Intern. Med. 156, 1143-1148 (1996).

57. Suarez, A. et al. Impaired tyrosine-kinase activity of muscle insulin receptors from hypomagnesaemic rats. Diabetologia 38, 1262-1270 (1995).

58. Rude, R. K. Magnesium deficiency and diabetes mellitus: Causes and effects. Postgrad. Med. 92, 217-224 (1992).

59. Vitale, J. Magnesium deficiency and cardiovascular disease. Lancet 340, 1224-1225 (1992).

\section{Author contributions}

S.E.M. and S.M.G. designed the research; S.E.M., S.M.G., and H.M. conductedthe research; S.E.M. and H.M. performed statistical analysis; S.E.M., S.M.G. and A.H. wrote the paper;S.E.B. and H.M. contributed to the revision of the manuscript, H.M. had primary responsibility forfinal content. All authors read and approved the final manuscript.

\section{Competing interests}

The authors declare no competing interests.

Additional information

Supplementary Information The online version contains supplementary material available at https://doi.org/ 10.1038/s41598-021-03915-3.

Correspondence and requests for materials should be addressed to H.M.

Reprints and permissions information is available at www.nature.com/reprints.

Publisher's note Springer Nature remains neutral with regard to jurisdictional claims in published maps and institutional affiliations.

(c) (i) Open Access This article is licensed under a Creative Commons Attribution 4.0 International cc) License, which permits use, sharing, adaptation, distribution and reproduction in any medium or format, as long as you give appropriate credit to the original author(s) and the source, provide a link to the Creative Commons licence, and indicate if changes were made. The images or other third party material in this article are included in the article's Creative Commons licence, unless indicated otherwise in a credit line to the material. If material is not included in the article's Creative Commons licence and your intended use is not permitted by statutory regulation or exceeds the permitted use, you will need to obtain permission directly from the copyright holder. To view a copy of this licence, visit http://creativecommons.org/licenses/by/4.0/.

(C) The Author(s) 2021 